\title{
A NEW CRITERION FOR UNIFORM DISTRIBUTION
}

\author{
P. E. O'NEIL ${ }^{1}$
}

1. A criterion for uniform distribution. Let $[a, b]$ be an interval which is a subset of the interval $[0,1]$. We denote this interval by $P$ and say it has length $p=b-a$. Let $\chi_{1}, \chi_{2}, \cdots, \chi_{k}, \cdots$ be a finite or infinite sequence of points in the interval $[0,1]$. For any integer $n>0$, we say that the ratio measure of $P, R_{n}(P)$, is the number of points in the finite partial sequence $\chi_{1}, \chi_{2}, \cdots, \chi_{n}$ which lie in the interval $P$ divided by the number $n$ of points in the sequence.

Definition. The infinite sequence $\chi_{1}, \chi_{2}, \cdots, \chi_{k}, \cdots$ is said to be "uniformly distributed" if for any $p$-length interval $P$, a subset of $[0,1]$,

$$
\operatorname{Lim}_{n \rightarrow \infty} R_{n}(P)=p
$$

A necessary and sufficient condition that a sequence be uniformly distributed already exists [1]. This paper offers a new, elementary and perhaps more intuitive condition.

Definition. Let there be given a set of points $\left\{a_{1}, a_{2}, \cdots, a_{n}\right\}$ in the interval $[0,1], a_{1}<a_{2}<\cdots<a_{n}$. The set is said to be an "almost-arithmetic progression- $(\delta, \epsilon)$ " if the following properties hold: There exists an $\eta \leqq \epsilon$ such that

(1.1) $a_{1}$ lies in the interval $[0, \eta+\delta \eta]$,

(1.2) for $k=1,2, \cdots, n-1$, the point $a_{k+1}$ lies in the interval $\left[a_{k}+\eta-\delta \eta, a_{k}+\eta+\delta \eta\right]$,

(1.3) $a_{n}$ lies in the interval $[1-\eta-\delta \eta, 1]$.

An almost-arithmetic progression- $(\delta, \epsilon)$ will be referred to as an almost-arithmetic progression when there can be no confusion, and may be abbreviated as an "a.-a.p.".

The motivation for the definition of an almost-arithmetic progres$\operatorname{sion}-(\delta, \epsilon)$ is in the following lemma.

Lemma 1.1. Given any $\epsilon^{\prime \prime}$, there exist $\delta, \epsilon$ such that for any interval $P$ of length $p$ and any almost-arithmetic progression- $(\delta, \epsilon), a_{1}, a_{2}, \cdots, a_{n}$, the ratio measure $R_{n}(P)$ falls within $\epsilon^{\prime \prime}$ of $p$.

Proof. The proof is elementary and will be omitted.

Received by the editors April 20, 1967.

1 I am indebted to the referee for his valuable comments and suggestions. 
THEOREM 1.2. The infinite sequence of points $\chi_{1}, \chi_{2}, \cdots, \chi_{n}, \cdots$, in $[0,1]$, is uniformly distributed iff the following condition holds: Given $\delta, \epsilon$, and $\epsilon^{\prime}$ all greater than zero, there exists an $N$ such that for $n>N$, the partial sequence $\chi_{1}, \chi_{2}, \cdots, \chi_{n}$ may be partitioned into a set of disjoint almost-arithmetic progressions- $(\delta, \epsilon)$ with at most $n_{0}$ points left over, $n_{0}<\epsilon^{\prime} n$.

Proof of Sufficiency. Given $\epsilon^{\prime \prime}$, choose $\delta$ and $\epsilon$ as in Lemma 1.1. Take any $\epsilon^{\prime}$ and find $N$. If $n>N$, we know that $n-n_{0}$ points of the sequence $\chi_{1}, \chi_{2}, \cdots, \chi_{n}$ are partitioned into almost-arithmetic progressions- $(\delta, \epsilon)$. For any interval $P$ in $[0,1]$, each of these a.-a.p's gives a ratio measure $R_{v}(P)$ which falls within $\epsilon^{\prime \prime}$ of $p$ by Lemma 1.1, $v$ being the number of points in the a.-a.p. If we consider the ratio measure $R_{n-n_{0}}(P)$ using all points which occur in a.-a.p's, the quantity will be a convex average of each single ratio measure of a particular a.-a.p. and will therefore fall within $\epsilon^{\prime \prime}$ of $p$. The remaining $n_{0}$ points can only serve to increase the difference of the ratio measure, $R_{n}(P)$, from $p$ by the quantity $\epsilon^{\prime}$.

Therefore we see

$$
\left|R_{n}(P)-p\right|<\epsilon^{\prime}+\epsilon^{\prime \prime}
$$

and since the right-hand side of (1.4) may be made as small as we wish for $N$ sufficiently large, the sequence is uniformly distributed.

ProOF OF NECESSITY. Let $\chi_{1}, \chi_{2}, \cdots, \chi_{k}, \cdots$ be an infinite sequence, uniformly distributed on $[0,1]$. We will attempt to show that the proposed necessary condition holds. Choose $\delta, \epsilon$, and $\epsilon^{\prime}$, where we take $\delta$ to be $1 / m$ for some integer $m$ without loss of generality. Now divide the interval $[0,1]$ into intervals $A_{1}, A_{2}, \cdots, A_{l}$, each of length $(\delta \eta) / 2$ which together cover the interval $[0,1]$ and overlap only at endpoints.

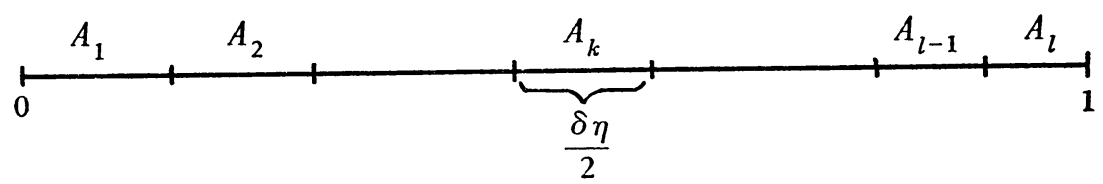

We have chosen $\eta<\epsilon$ so that there will be an integral number of such intervals $A_{i}$. That is $2 / \delta \cdot \eta=l$, an integer.

Now since $\left\{\chi_{k}\right\}$ is uniformly distributed, given $\epsilon_{0}$, for all $i$, we may choose an $N_{i}$, so that if $n \geqq N_{i}$ we always have

$$
\delta \eta / 2-\epsilon_{0} \leqq R_{n}\left(A_{i}\right) \leqq \delta \eta / 2+\epsilon_{0} .
$$

Let $N=\max _{i=1}, \cdots, l N_{i}$. We now have that for $n \geqq N$, equation (1.5) holds for all $i=1, \cdots, l$. 
Fix $n \geqq N$ and let $A_{k}$ be the interval containing the fewest points of the sequence $\left\{\chi_{i}\right\}$ of all the intervals. Certainly it contains at least $\left(\delta \eta / 2-\epsilon_{0}\right) \cdot n$ points. Now go through each of the other intervals, removing points of the sequence $\left\{\chi_{i}\right\}$ from them until they each contain the same number of points as the interval $A_{k}$ ( $A_{k}$ is not touched). The total number of points which has been removed is $n_{0}$ and is obviously bounded above by $(l-1) \cdot\left(2 \epsilon_{0} \cdot n\right)$. By making $\epsilon_{0}$ sufficiently small, we may be assured that $n_{0} \leqq \epsilon^{\prime} n$.

The points which remain in the intervals $A_{1}, A_{2}, \cdots, A_{l}$ may now be partitioned into almost-arithmetic progressions- $(\delta, \epsilon)$, which will conclude the proof.

First break the intervals into different conjugate classes mod $2 / \delta$ as regards their subscripts $(2 / \delta$ is an integer). We now have sequences of intervals of the form:

$$
\begin{gathered}
A_{1}, A_{2 / \delta+1}, A_{4 / \delta+1}, \cdots, A_{2 / \eta \delta-2 / \delta+1} \\
A_{2}, A_{2 / \delta+2}, A_{4 / \delta+2}, \cdots, A_{2 / \eta \delta-2 / \delta+2} \\
. . . . . . . . . . . ~ \\
A_{2 / \delta}, A_{4 / \delta}, A_{6 / \delta}, \cdots, A_{2 / \eta \delta}
\end{gathered}
$$

It is an easy exercise to verify that if one element is chosen out of each interval in a row of the above array, the set obtained will be an almost-arithmetic progression- $(\delta, \epsilon)$. Since each of the intervals contains the same number of points of $\left\{\chi_{i}\right\}$, these points may indeed be partitioned into almost-arithmetic progressions- $(\delta, \epsilon)$, and the proof is complete.

2. Applications. It is not at first apparent that the above condition may be readily applied to show that specific sequences are uniformly distributed, but in fact the application is usually obvious and supplies an intuitive insight into the reason that the sequence is uniformly distributed.

EXAMPLE 1. Given an irrational number $a$, show that the sequence $\chi_{k}=a \cdot k-[a \cdot k]$ is uniformly distributed.

Proof. Let $\epsilon, \delta$, and $\epsilon^{\prime}$ be given. Let $m$ be chosen so that $1 / m<\epsilon$. If one looks at the first $m+1$ elements of the sequence $\chi_{i}$, it is obvious that two of them, say $\chi_{s}$ and $\chi_{t}$, must lie within a distance of $1 / m$ of one another. Let us say $\mathrm{s}<t, t-s=l$ and $\chi_{t}$ is to the right of $\chi_{s}$.

Now break up the elements of the sequence $\chi_{1}, \cdots, \chi_{n}$ into conjugate classes $\bmod l$ with respect to their subscripts: 


$$
\begin{aligned}
& \chi_{1} \quad \chi_{l+1} \cdots \chi_{m_{1} l+1} \\
& \chi_{2} \quad \chi_{l+2} \cdots \chi_{m_{2} l+2} \\
& \chi_{l} \quad \chi_{2 l} \quad \cdots \chi_{m l \cdot l}
\end{aligned}
$$

Looking at each row in the above array, it is clear that the first element may fall somewhere in the interval $[0,1]$, the second a distance $\eta \leqq 1 / m<\epsilon$ to the right until an element comes within $\eta$ of 1 . The elements so far have not gone into an a.-a.p. But the next element will fall within $\eta$ of 0 ; successive elements will move to the right in steps of $\eta$ until the last element is within $\eta$ of 1 . The sequence which started at zero is an almost-arithmetic progression- $(\delta, \epsilon)$. (In fact, $\delta$ is zero; it is a true arithmetic progression.) The next element of the sequence will be the first of a new a.-a.p. This process will continue until the sequence ends, perhaps stopping at a distance greater than $\eta$ from 1 . Now those elements which did not fall in an a.-a.p. may be bounded above in number, with the bound depending only on $a$ and the integer $m$. Multiplying by $l$, we will have an upper bound $B$ not depending on $n$ (the number of points in the partial sequence) for the number of points which do not fall in a.-a.p's, i.e., $n_{0} \leqq B$. We may increase $n$ until $B<\epsilon^{\prime} n$ and we are finished.

It is obvious that only crude approximations were used here, and it would seem that good lower bounds for the actual speed of convergence of the ratio measure $R_{n}$ to the measure of an arbitrary interval $P$ may be achieved for specific irrationals $a$ by finer applications of this method.

Example 2 (From Pólya And Szegö [2]). $\chi_{k}=a k^{\sigma}-\left[a k^{\sigma}\right], a>0$, $0<\sigma<1$ is uniformly distributed. It is left as an easy exercise that if $\delta, \epsilon$ are given, we may choose $N^{\prime}$ such that for $n \geqq N^{\prime}$, then for all $k$, $n \leqq a k^{\sigma} \leqq n+1$, the set of points $\chi_{k}$ thus chosen form an almostarithmetic progression- $(\delta, \epsilon)$. All the points $\chi_{k}$ such that $a k^{\sigma}<N^{\prime}$ may be thrown away, together with all the points which overlap at the end so long as $N-N^{\prime} \gg N^{\prime}$ ( $N$ is the $N$ of the criterion).

ExAmple 3 (From Pólya ANd Szegö). $\chi_{n}=a(\log n)^{\sigma}-\left[a(\log n)^{\sigma}\right]$, $a>0, \sigma>1$. We may use the same method as Example 2. After some experience with the criterion, the check of uniform distribution becomes a simple mental calculation for sequences of this type. Note that if $\sigma=1$ in the above example, uniform distribution does not hold. The intuition supplied by this criterion immediately suggests a proof of the fact. 


\section{BIBLIOGRAPHY}

1. J. W. S. Cassels, An introduction to diophantine approximation, Cambridge Tracts in Math. and Math. Phys., no. 45, Cambridge Univ. Press, New York, 1957, Ch. IV, Sec. IV.

2. G. Pólya and G. Szegö, Aufgaben und Lehrsätze auf der Analysis, 3rd ed., Die Grundlehren der math. Wissenschaften, Bände 19, 20, Springer-Verlag, New York, 1964.

ibm Cambridge Scientific Center, Cambridge, Massachusetts 